\title{
Different Brains Process Numbers Differently: Structural Bases of Individual Differences in Spatial and Nonspatial Number Representations
}

\author{
Florian Krause ${ }^{1}$, Oliver Lindemann ${ }^{2}$, Ivan Toni ${ }^{1}$, \\ and Harold Bekkering ${ }^{1}$
}

\begin{abstract}
A dominant hypothesis on how the brain processes numerical size proposes a spatial representation of numbers as positions on a "mental number line." An alternative hypothesis considers numbers as elements of a generalized representation of sensorimotor-related magnitude, which is not obligatorily spatial. Here we show that individuals' relative use of spatial and nonspatial representations has a cerebral counterpart in the structural organization of the posterior parietal cortex. Interindividual variability in the linkage between numbers and spatial responses (faster left responses to small numbers and
\end{abstract}

right responses to large numbers; spatial-numerical association of response codes effect) correlated with variations in gray matter volume around the right precuneus. Conversely, differences in the disposition to link numbers to force production (faster soft responses to small numbers and hard responses to large numbers) were related to gray matter volume in the left angular gyrus. This finding suggests that numerical cognition relies on multiple mental representations of analogue magnitude using different neural implementations that are linked to individual traits.

\section{INTRODUCTION}

Dealing with numerical information is an integral part of our modern society. Numbers occur throughout all aspects of everyday life; they depict information about prices and values and allow us to count occurrences and entities. During a single day, we probably process several thousand numbers (Butterworth, 1999). Yet, our ability to deal with them varies greatly across individuals (Butterworth, 2010; Adams, 2007). It is therefore important to understand how individuals represent numbers and how their brains process this information. The most influential model of number processing, the triple-code model, dissociates between three different numerical representations: an Arabic code for digit processing, a verbal code for retrieval of arithmetic facts and verbal counting, and an analogue magnitude code for the processing of numerical size (Dehaene, 1992). Whereas the first two representations are notation and modality dependent, an analogue magnitude representation is thought to be abstract in nature and is thus assumed to be independent of notation and modality (Cohen Kadosh \& Walsh, 2009). The current study seeks to investigate two different manifestations of this analogue magnitude representation of numerical size.

Over the last decades, several studies on number cognition have provided abundant empirical support for the

${ }^{1}$ Radboud University Nijmegen, ${ }^{2}$ University of Potsdam

hypothesis that numerical size is spatially represented in the brain (Dehaene, 2009; de Hevia, Vallar, \& Girelli, 2008; Hubbard, Piazza, Pinel, \& Dehaene, 2005). This hypothesis assumes that we derive the size of a number from its position on an ordered "mental number line" on which small numbers are represented on one side and large numbers on the other (Moyer \& Landauer, 1967). For instance, the so-called effect of spatial-numerical association of response codes (SNARC) shows a linkage between numerical information and spatial responses (Dehaene, Bossini, \& Giraux, 1993). When participants are asked to judge the parity of Arabic digits between 1 and 9 by a left or right response, the numerical size of the digit interferes with the execution of the spatial responses, with faster left responses to small numbers and faster right responses to large numbers. This spatial number-response interference effect has been interpreted as evidence for a shared representation of spatial response codes and the ordinal position of the number representation in mental space.

More recently, the assumption that spatial codes become obligatorily activated when processing numerical size has been questioned by several authors (e.g., Santens \& Gevers, 2008; Fischer, 2006). It has been argued instead that information about numerical size is mapped onto representations of other size-related sensorimotor information within a system that processes generalized analogue magnitude (Walsh, 2003). According to this 
hypothesis, number meaning is conceptualized by recruiting the same mechanisms that allow us to experience and control other behaviorally relevant magnitudes in daily life. Evidence for this notion comes from several studies showing associations between numbers and other types of magnitude information in action and perception, like physical size (Tzelgov, Meyer, \& Henik, 1992), temporal duration (Oliveri et al., 2008), grip aperture (Lindemann, Abolafia, Girardi, \& Bekkering, 2007), object graspability (Badets, Andres, Di Luca, \& Pesenti, 2007), and tactile sensation (Krause, Bekkering, \& Lindemann, 2013). Crucially, the associated sensorimotor magnitudes can be entirely nonspatial in nature. For instance, a link between numerical information and force production has been reported (Vierck \& Kiesel, 2010), which we will refer to as forcenumerical association of response codes (FoNARC). When participants are asked to judge the parity of Arabic digits between 1 and 9 by a soft or hard response on a single button, the numerical size of the digit interferes with the execution of force responses, with faster soft responses to small numbers and faster hard responses to large numbers. Importantly, the procedure to quantify a FoNARC effect is identical to the procedure to quantify a SNARC effect except that required motor responses for the latency measurement do not differ spatially. Because of the homogeneity of all spatial response components, it can be excluded that the number-response interference effect observed under these condition is driven by a spatial representation of numbers on a mental number line. The observation of a FoNARC effect consequently has to be interpreted as a within-magnitude inference between numerical information and the control of motor force, which in turn suggests the existence of nonspatial sensorimotorrelated representations of numbers.

With the apparent coexistence of both spatial and nonspatial representations of numerical size, the question arises in which way the two representations contribute to numerical cognition. It has been suggested that multiple representations of the same numerical information rely on different neural implementations and that the weights of their contribution are simply determined by the requirements of the situation or task at hand (Dehaene, Piazza, Pinel, \& Cohen, 2003). A numerical task with a spatial component would lead to a stronger activation of posterior superior parietal lobe. In contrast, a number task without any spatial component (e.g., force production) is expected to engage primarily inferior parietal regions (cf. Dehaene et al., 2003).

However, task demands might not be the only factor to determine how numerical information are processed. For instance, the general disposition to associate numbers with space has been shown to vary strongly between individuals (for a review, see Wood, Nuerk, \& Willmes, 2008) and might even depend on personal preferences to code numerical information (Fischer, 2006). The same might hold for linking numbers to nonspatial sensorimotor-related magnitude, as this disposition might be related to the individual's bodily competence and experience of dealing with magnitudes and sizes in everyday life (Lindemann, Rueschemeyer, \& Bekkering, 2009; see also Barsalou, 2008). Here we assess whether those interindividual differences reflect stable individual traits, rather than stochastic noise or task demands.

This issue was addressed by combining a double dissociation approach with the method of voxel-based morphometry (VBM; Ashburner \& Friston, 2000). The rationale of this approach is to isolate differential structural variances across two behavioral indexes of numerical cognition, intrinsically controlling for confounds correlated with both indexes. We tested whether interindividual variation in anatomical brain structure explains individual differences in spatial (SNARC) and nonspatial (FoNARC) number-response interference effects-reflecting a spatial and nonspatial representation of numerical size, respectively. VBM was used to measure variability in local gray matter volume in the posterior parietal cortex, a site consistently associated with numerical representations (see Arsalidou \& Taylor, 2011, for a review). fMRI was used to map the spatial distribution of task-related activity across the posterior parietal cortex.

\section{METHODS}

\section{Participants}

A total of 33 students (20 women) between 18 and 34 years (mean age $=21.33$ years, $S D=3.28$ years) participated in the experiment in return for $€ 20$ or course credits. All of them had normal or corrected-to-normal vision and were of general health, with no known neurological or psychological disorders. The study was approved by the local ethics committee, and participants gave their written consent before the experimental procedure.

\section{Setup}

Stimuli consisted of the Arabic digits 1-9, except 5, depicted in white color (visual angle: $\sim 1.26^{\circ}$ vertical and $\sim 0.53^{\circ}$ horizontal) centrally on a black background. Participants viewed the screen via a mirror attached to the MR scanner's receiver head coil.

Responses were recorded using MR-compatible button boxes with either spatially arranged buttons that had to be pressed with the right index and right middle finger or with a single isometric force-transducer button, which had to be pressed with the right thumb. The forcesensitive button box was a cylinder grasped between the thumb and the remaining fingers.

\section{Procedure}

The data collection was done in the context of a larger fMRI study and was thus performed while participants were lying inside the MR scanner. Participants were 
engaged in two consecutive number parity judgment tasks in which the presented digits had to be classified as odd or even. Importantly, both tasks differed only in the required responses. In the spatial task, number parity had to be indicated by a right index finger ("left") or middle finger ("right") response. That is, each response involved the flexion and extension of either one of the two fingers. In the nonspatial task, responses were given with the right thumb and involved applying either a small force (>500 cN, "soft" responses) or a large force (>2500 cN, "hard" responses).

Each trial started with the presentation of a white fixation cross for $500 \mathrm{msec}$, followed by the target stimulus. Participants had to respond within 1000 msec after stimulus presentation. If it took them too long to respond or their response was incorrect, an auditory error signal was played back to them via headphones. After the response was given, a dark gray fixation cross was presented for a variable time between 2000 and $4000 \mathrm{msec}$, before the next trial started.

Before the actual experiment, participants were given verbal instructions and practiced the task for about 5 min outside the MR scanner. The response mapping to indicate the parity (i.e., left or right response for odd numbers and soft or hard response for odd numbers) was reversed in the middle of each task block. The order of mappings and the order of the spatial and nonspatial task were balanced between participants. Eighteen participants performed 320 trials, and 15 participants performed 288 trials. The order of trials was randomized.

\section{MRI Data Acquisition}

For each participant, a high-resolution anatomical MR image was recorded using a T1-weighted MP-RAGE sequence with a GRAPPA acceleration factor of 2 (repetition time/echo time $=2300 / 3.03 \mathrm{msec}$, voxel size $=1 \times$ $1 \times 1 \mathrm{~mm})$. Anatomical images were recorded directly after both tasks were conducted. Because of technical problems, MR images of two participants were acquired 12 and 5 weeks after the behavioral test, respectively. For one participant, a 7-months-older MR image was used. All images were recorded on the same 3-T Siemens Magneton Trio MR scanner (Siemens, Erlangen, Germany) in combination with the same 32-channel receiver head coil.

Functional images were acquired using a multi-echo gradient-echo planar $\mathrm{T} 2 *$-weighted sequence sensitive to BOLD contrast (repetition time $=2390 \mathrm{msec}$; echo time $=$ 9.4, 21.2, 33.0, 45.0 and $56.0 \mathrm{msec}$; flip angle $=90^{\circ}$; field of view $=224 \times 224 \mathrm{~mm}$; number of slices $=31$; slice thickness $=3 \mathrm{~mm}$; resolution $=3.5 \times 3.5 \times 3.0 \mathrm{~mm}$ ).

\section{Behavioral Data Analysis}

The behavioral data of each participant were analyzed separately for the spatial and the nonspatial task to estimate effect sizes for both, a SNARC effect in the spatial task as well as a FoNARC effect in the nonspatial task. Only trials with correct parity judgments within 1000 msec were included in the analysis. Effect sizes were calculated as suggested by Fias, Brysbaert, Geypens, and Ydewalle (1996). First, the difference in the median RTs between left and right responses (spatial task) and soft and hard responses (nonspatial task) was calculated for each presented digit. Then, individual linear regressions between these RT differences and the digits were calculated. The resulting regression coefficients were used to characterize the size of the SNARC or FoNARC effect in each participant.

\section{VBM Analysis}

MR image preprocessing and statistical testing was done using Statistical Parametric Mapping 8 (SPM8, www.fil. ion.ucl.ac.uk/spm) and the integrated DARTEL toolbox (Ashburner, 2007).

Each anatomical image was segmented into gray and white matter images and resampled to $1.5 \mathrm{~mm}$ isotropic resolution. Afterwards, nonlinear deformations for warping all gray and white matter images to each other were determined by iterative template creation (seven steps; Ashburner, 2007). Modulated warped gray matter images were created by smoothing with a Gaussian kernel of $10 \mathrm{~mm}$ and normalizing to the Montreal Neurology Institute (MNI) coordinate space.

An anatomical mask was created using the SPM8 Anatomy Toolbox (Eickhoff, Stephan, Mohlberg, \& Grefkes, 2005), including portions of the superior parietal cortex (areas 7A, 7PC, 7M, 7P; Scheperjans, Eickhoff, et al., 2008; Scheperjans, Hermann, et al., 2008), the inferior parietal cortex (areas PFop, PFt, PF, PFm, PFcm, PGa, PGp; Caspers et al., 2006, 2008), as well as the intraparietal sulcus (areas hIP1, hIP2, hIP3; Scheperjans, Eickhoff, et al., 2008; Scheperjans, Hermann, et al., 2008; Choi et al., 2006).

This anatomical mask, based on regions previously involved in numerical cognition (Wu et al., 2009; Cohen Kadosh, Lammertyn, \& Izard, 2008), was combined with a functional mask including posterior parietal voxels activated during either one of the experimental tasks (see fMRI analysis below).

The preprocessed images entered a multiple regression general linear model (GLM) with SNARC and FoNARC effect size estimates as regressors of interest. Two additional covariates were added to the GLM: median overall RTs, aggregated over both tasks, to control for general performance differences between participants, and total intracranial volume, to control for general overall size differences of gray matter, white matter, and cerebrospinal fluid (Good et al., 2001).

The statistical threshold was $p<.05$ at voxel level, corrected for multiple comparisons by means of the familywise error (FWE). 


\section{fMRI Analysis}

Functional image preprocessing and statistical testing were done using SPM8 (www.fil.ion.ucl.ac.uk/spm).

For each volume, the five multi-echo images were combined into a T2*-weighted average image (Poser, Versluis, Hoogduin, \& Norris, 2006). All weighted average images were spatially realigned to the first image and corrected for differences in slice time acquisition. The T1-weighted anatomical image was coregistered with the mean functional image, segmented and normalized to the MNI standard space, and resampled to a $2 \times$ $2 \times 2 \mathrm{~mm}$ resolution. The resulting normalization parameters were applied to the functional images, which were subsequently spatially smoothed using a Gaussian kernel of $8 \mathrm{~mm}$.

The preprocessed images entered a GLM with four sessions, describing two response mappings for each of the two experimental tasks. For each session, task effects were modeled using a combination of compatible and incompatible trials for four groups of numerical stimuli $(1,2 ; 3,4 ; 6,7 ; 8,9)$, resulting in eight regressors, each describing the onset of the response to the stimulus. An additional regressor was used to model erroneous responses. All task-related regressors were convolved with a hemodynamic response function. Three translational and three rotational head motion parameters and their first and second derivative, resulting in 18 regressors, were added as covariates.

To capture posterior parietal voxels activated during the experimental tasks, the $t$ contrast of each task compared with implicit baseline was evaluated for the whole brain on the group level (thresholded at 0.05, uncorrected). The union of the results of both contrasts, restricted to the entire posterior parietal cortex (i.e., areas $5 \mathrm{~L}, 5 \mathrm{M}$, 5Ci, 7A, 7PC, 7M, 7P, PFop, PFt, PF, PFm, PFcm, PGa, PGp, hIP1, hIP2, hIP3), served as a functional mask (see VBM analysis above).

We also assessed whether the regions showing structural variations as a function of SNARC/FoNARC performance were also functionally engaged in performance of those tasks. That is, we tested whether the fMRI data showed increased BOLD signal $(p<.01$, FWE-corrected for search volume) within a search volume defined by two spherical VOIs centered on the two local maxima of the VBM analyses, with a radius matched to the FWHM of the VBM results $(10 \mathrm{~mm})$.

\section{RESULTS}

\section{Behavioral Results}

On average, participants made $5 \%$ errors in the spatial task and $10 \%$ errors in the nonspatial task. The average
Figure 1. Relative changes in gray matter volume in the posterior parietal cortex, related to spatial and nonspatial representations of numerical size. Individuals' disposition to associate numbers with spatial responses predicts local gray matter volume in right precuneus (blue). The disposition to link numbers to force production predicts gray matter volume in left angular gyrus (red). Thresholded at $p<.001$, uncorrected, for illustrative purposes.

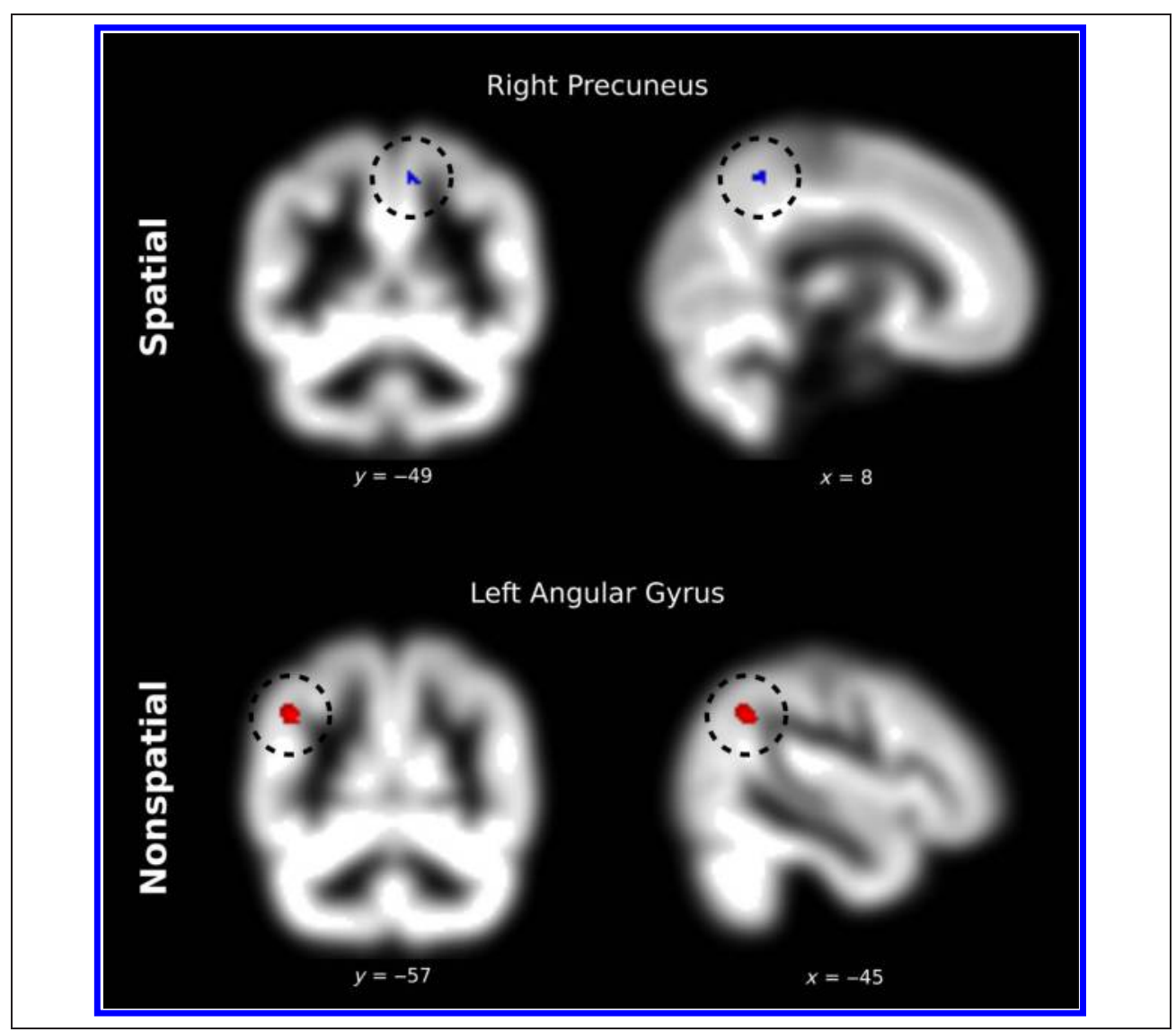


Figure 2. Correlations between gray matter volume and number-response interference effects, corrected for average RT and total intracranial volume, demonstrating a double dissociation between brain regions and representations of numerical size. Significant correlations (as revealed by the multiple regression analysis) are plotted with a continuous regression line.

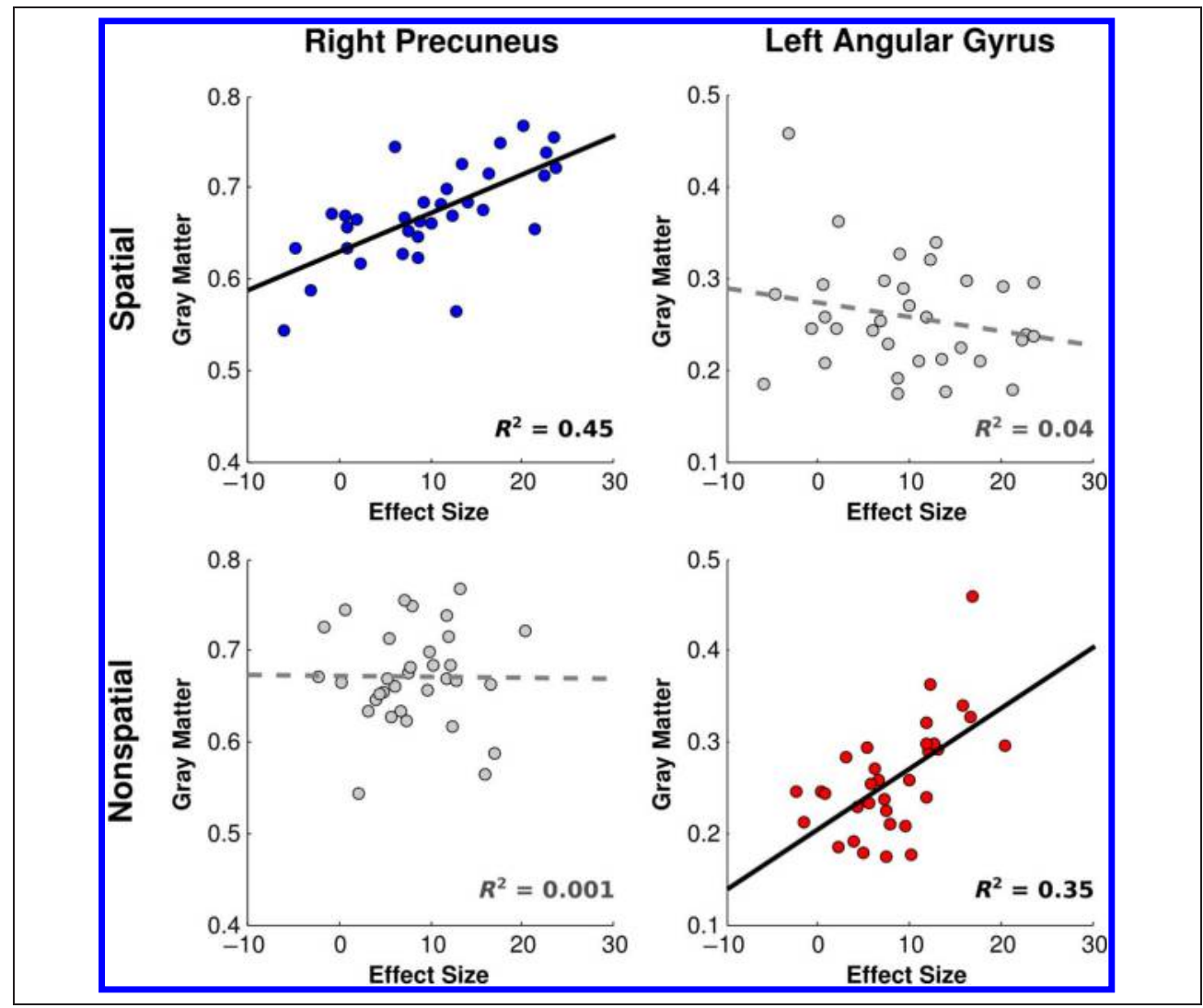

RTs were $539 \mathrm{msec}(S D=60 \mathrm{msec})$ and $585 \mathrm{msec}(S D=$ $62 \mathrm{msec}$ ) for the spatial task and nonspatial task, respectively. SNARC effect sizes of all participants differed significantly from zero, $t(32)=6.70, p<.001$, as did FoNARC effect sizes, $t(32)=8.61, p<.001$. There was a weak, but nonsignificant, positive correlation between the individual SNARC and FoNARC effect sizes, $r=$ $0.31, p=.07$. Median overall RTs correlated with the size of the SNARC effect, $r=0.36, p<.05$, and were therefore included as an additional covariate in the GLM for the VBM analysis (see Methods). Importantly, there were no correlations between age and SNARC effect sizes, $r=-0.10, p=.57$, age and FoNARC effect sizes, $r=0.03, p=.88$, or gender and SNARC effect sizes, $r=$ $0.11, p=.55$, and gender and FoNARC effect sizes, $r=$ $0.16, p=.37$. Therefore, and because any shared variance with age and gender is common to both regressors of interest in the GLM, age and gender were not added as explicit covariates into the VBM analysis.

\section{VBM Results}

Figure 1 shows the main findings of the VBM analysis (thresholded at 0.001, uncorrected, for illustrative purposes). The multiple regression analysis on the posterior parietal cortex revealed that SNARC effect size predicted local relative gray matter volume in the right precuneus (area 5M; peak at MNI coordinates $x=7.5, y=-49.5$, $\left.z=52.5 ; t(28)=4.97, Z=4.17, p_{\mathrm{FWE}}<.05\right)$. The stronger the individual SNARC effect (i.e., the disposition to associate numbers with a spatial response), the more relative gray matter was present in this particular region. Furthermore, FoNARC effect size predicted local relative gray matter volume in the left angular gyrus (area PGa; peak at MNI coordinates $x=-45, y=-57, z=37.5), t(28)=5.37$, $Z=4.42, p_{\mathrm{FWE}}<.05$. The stronger the FoNARC effect (i.e., the disposition to link numbers to force production), the more relative gray matter in this region of the individual's brain. Figure 2 illustrates the differential correlations between gray matter volume in each of the regions and number response interference effects, corrected for average RT and total intracranial volume. Importantly, gray matter volume in right precuneus correlated significantly more with the spatial than with the nonspatial number response interference effect, $Z=3.55, p<.01$, whereas gray matter volume in left angular gyrus correlated significantly less with the spatial than with the nonspatial number response interference effect, $Z=-3.89$, $p<$ .01 , demonstrating a double dissociation between the behavioral and the structural cerebral effects.

\section{fMRI Results}

SVC analysis on the regions identified in the VBM analysis revealed a significant activation of right precuneus during both the spatial task, $t(32)=5.31, Z=4.47, p_{\mathrm{FWE}}<.01$, 
and the nonspatial task, $t(32)=4.57, Z=3.98, p_{\mathrm{FWE}}<$ .01. Likewise, left angular gyrus was significantly activated during the spatial task, $t(32)=5.05, Z=4.30, p_{\mathrm{FWE}}<$ .01 , as well as during the nonspatial task, $t(32)=6.97$, $Z=5.40, p_{\mathrm{FWE}}<.01$.

\section{DISCUSSION}

This study provides evidence for a contribution of both spatial and nonspatial representations of numerical size when processing Arabic digits and demonstrates that the weights of this contribution rely on stable individual traits. We show that interindividual differences in the dispositions to link numbers to either space or nonspatial sensorimotor magnitude can be directly related to structural variance in two distinct regions in the superior and inferior posterior parietal lobes.

\section{Structural Bases of Spatial and Nonspatial Representations of Numerical Size}

There was a relation between the strength of the SNARC effect and the structure of a parietal region (area $5 \mathrm{~m}$ ) in the right precuneus. Increased gray matter in this region predicted stronger interference of numerical size with spatial responses, but not with the force of a response. Although little is known about the specific functionality of area $5 \mathrm{~m}$ in humans, its cytoarchitecture suggests that it is comparable with area PE in the macaque brain (Scheperjans, Grefkes, Palomero-Gallagher, Schleicher, \& Zilles, 2005). Macaque PE has been involved in somatosensory integration and in creating a spatial representation of limbs during movement (Bakola, Passarelli, Gamberini, Fattori, \& Galletti, 2013; Lacquaniti, Guigon, Bianchi, Ferraina, \& Caminiti, 1995; Jones, Coulter, \& Hendry, 1987; Mountcastle, Lynch, Georgopoulos, Sakata, \& Acuna, 1975). In humans, the right precuneus has repeatedly been shown to be important for spatial processing, such as shifting attention in visual space or visual imagery (for a review, see Cavanna \& Trimble, 2006), but not in the processing of numerical magnitude information (for a review, see Dehaene et al., 2003). In fact, the present findings indicate that this parietal region is involved in the spatial representation of numbers, suggesting that the association between numbers and space is closer to general cognitive-spatial processing than to numerical magnitude per se.

The strength of mapping numbers to motor force (FoNARC effect) correlated with the structure of the left angular gyrus. Increased gray matter in this region predicted stronger interference of numerical size with the force of a response, but not with the laterality of a response. In contrast to the precuneus, the angular gyrus - the left side in particular-has been consistently related to the processing of numerical information (Arsalidou \& Taylor, 2011). For instance, a lesion in the left angular gyrus can lead to arithmetical deficits (Gerstman Syndrome; Gerstmann, 1940). Dehaene et al. (2003) concluded that the left angular gyrus is involved in the retrieval of linguistic arithmetic facts from memory. Evidence for this notion comes, for instance, from neuroimaging studies showing that activity in this region is modulated by arithmetic training (Ischebeck et al., 2006; Delazer et al., 2003). Further support for memorybased processes is provided by the observation that neuronal activity in angular gyrus is higher when arithmetic problems are solved by fact retrieval, compared with calculations (Grabner et al., 2009). In contrast, it has been argued by several authors that the angular gyrus is involved in the processing of number symbols and their numerical magnitude information (Rusconi, Walsh, \& Butterworth, 2005; Göbel, Walsh, \& Rushworth, 2001). For instance, TMS over angular gyrus is known to disrupt parity as well as magnitude comparisons (Rusconi et al., 2005). An involvement of the left angular gyrus has been furthermore demonstrated while processing numerical symbols in the absence of any arithmetic demands (Price \& Ansari, 2011; Holloway, Price, \& Ansari, 2010). Ansari (2008) hypothesized therefore that the left angular gyrus mediates the mapping of numerical symbols onto magnitude representations. The present finding now adds further empirical evidence for this idea and demonstrates an involvement of the left angular gyrus in a nonlinguistic and nonspatial analogue representation of numerical size. We therefore interpret our findings as support for the notion that this region is involved in the mapping of number symbols onto magnitude information.

\section{Spatial and Nonspatial Number-Response Interference Effects}

The current findings shed new light on the nature of spatial and nonspatial number-response interferences effects and might also have practical implications for investigating numerical representations. This study is one of the first to demonstrate a direct brain correlate of the well-known association between numbers and spatial responses reflected by the SNARC effect. The results of a recent functional near-infrared spectroscopy study show a functional activation of bilateral intraparietal sulcus and left angular gyrus when participants are engaged in a spatial number response interference task (Cutini, Scarpa, Scatturin, Dell'acqua, \& Zorzi, 2012). This study now extends these findings and shows that the existing individual preferences in the association between numbers and space (e.g., Fischer, 2006) relate to structural variance in right precuneus. Importantly, the precuneus is known to be involved in the processing of various types of spatial information in different domains and modalities, but the brain region is typically not assumed to play any specific crucial role in the processing of numerical magnitude information. 
Taking into account the current finding and the general function of the precuneus for spatial processing, one might question whether spatial number-response interference effects are actually informing us about core mechanisms of number processing, rather than about the use of more general cognitive coding strategies. For instance, associations with space have also been observed for a variety of ordinal/sequential information like letters of the alphabet, months, or days of the week (Gevers, Reynvoet, \& Fias, 2003, 2004), suggesting that spatial associations are driven by any type of ordinal information and not specifically related to the representation of numerical magnitude. Our current data are in line with this view, as they suggest that the nature of the number-space mapping is not magnitude related, but related to a brain structure involved in general spatial processing. On the other hand, differences in the strength of the mapping between numbers and force production (FoNARC effect) were shown to be related to structural variance in a brain region known to be crucial for processing magnitude-related aspects of numbers. This emphasizes the importance of nonspatial magnitude representations and the suitability of using nonspatial magnitude-related number-response interference paradigms to investigate the mechanisms of numerical processing.

Both behavioral and VBM results of this study showed that the interindividual differences in the size of a SNARC and FoNARC effect are uncorrelated and independent of each other, which strongly suggests that the two effects reflect different aspects of the cognitive processing and representation of numerical information. The dissociation between spatial and nonspatial representations of numerical size might have relevant implications for education. Although a number line mapping seems to be a suitable tool for children to visualize numerical information (Fischer, Moeller, Bientzle, Cress, \& Nuerk, 2011), the success of this method might vary tremendously from child to child. If numerical representations vary strongly between individuals, as the current results suggest, identifying and supporting these differences might be educationally beneficial. However, at this point in time, the relation between differences in number representation and differences in number competence remain largely unclear. Although a relation between left angular gyrus activity and mathematical competence has recently been reported (Grabner et al., 2007), future research with a strong focus on interindividual differences in numerical skills will be needed to address this open question further.

\section{Interpretational Limitations of the Current Study}

The exact nature of the gray matter volumetric differences identified with VBM is still poorly understood, as they could be related to changes in neuropil, neuronal size, dendritic or axonal arborization, as well as cortical folding (Mechelli, Price, Friston, \& Ashburner, 2005). This complicates the interpretation of any VBM study with respect to linking structural variability to its underlying functionality. In the current study, this interpretational issue is somehow reduced by the observation that the task-related structural variability occurs in parietal regions functionally engaged during performance of those tasks. Although it is generally assumed that larger gray matter volume reflects enhanced neuronal processing (Kanai, Feilden, Firth, \& Rees, 2011; Mechelli et al., 2004; Maguire et al., 2000), future studies will need to detail the microstructural and computational mechanisms associated with the present findings.

The structural findings emerged from an analysis focused on the parietal lobe. The rationale of this choice was to include in the analysis posterior parietal areas previously shown to be involved in number processing (Wu et al., 2009; Cohen Kadosh et al., 2008) and active during performance of the tasks used in the current study. However, the structural changes reported here could also be observed in a whole-brain analysis (right precuneus: $Z=$ 4.40; left angular gyrus: $Z=4.42$ ).

It is unclear in how far the brain regions identified in the current study are bound to specific demands of the numerical tasks used. This is especially important for understanding the role of left angular gyrus during number processing. Here, force production was used as an instance of nonspatial sensorimotor magnitude. It remains to be seen whether the current observations generalize from the domain of force production to other nonspatial sensorimotor magnitudes.

\section{Conclusion}

Taken together, the current findings suggest that numerical cognition relies on multiple mental representations of analogue magnitude using distinct neural implementations that are linked to individual traits. We showed that the way we represent numerical size is not only dependent on situational requirements of a given task but also subject to interindividual differences. Importantly, these differences appear to be stable traits as they can be linked to distinct structural variance in the posterior parietal cortex. Our finding of individual traits stimulates new research to investigate whether these traits are innate ("nature") or the result of external factors and emerge only later during development ("nurture"; see also Dehaene, 1997)—a question whose answer will have wide implications for math education.

\section{Acknowledgments}

We would like to thank Pascal de Water and Paul Gaalman for technical support and assistance during data acquisition as well as Roi Cohen Kadosh for his valuable comments during an early data analysis stage.

Reprint requests should be sent to Florian Krause, P.O. Box 9104 , 6500 HE Nijmegen, The Netherlands, or via e-mail: f.krause@ donders.ru.nl. 


\section{REFERENCES}

Adams, J. W. (2007). Individual differences in mathematical ability: Genetic, cognitive and behavioural factors. Journal of Research in Special Educational Needs. 7, 97-103.

Ansari, D. (2008). Effects of development and enculturation on number representation in the brain. Nature Reviews Neuroscience, 9, 278-291.

Arsalidou, M., \& Taylor, M. J. (2011). Is $2+2=4$ ? Meta-analyses of brain areas needed for numbers and calculations. Neuroimage, 54, 2382-2393.

Ashburner, J. (2007). A fast diffeomorphic image registration algorithm. Neuroimage, 38, 95-113.

Ashburner, J., \& Friston, K. J. (2000). Voxel-based morphometry: The methods. Neuroimage, 11, 805-821.

Badets, A., Andres, M., Di Luca, S., \& Pesenti, M. (2007). Number magnitude potentiates action judgements. Experimental Brain Research, 180, 525-534.

Bakola, S., Passarelli, L., Gamberini, M., Fattori, P., \& Galletti, C. (2013). Cortical connectivity suggests a role in limb coordination for macaque area PE of the superior parietal cortex. The Iournal of Neuroscience, 33, 6648-6658.

Barsalou, L. W. (2008). Grounded cognition. Anmual Review of Psvchology, 59, 617-645.

Butterworth, B. (1999). The mathematical brain. London: Macmillan.

Butterworth, B. (2010). Foundational numerical capacities and the origins of dyscalculia. Trends in Cognitive Sciences, 14 , 534-541.

Caspers, S., Eickhoff, S., Geyer, S., Scheperjans, F., Mohlberg, H., Zilles, K., et al. (2008). The human inferior parietal lobule in stereotaxic space. Brain Structure and Function, 212, 481-495.

Caspers, S., Geyer, S., Schleicher, A., Mohlberg, H., Amunts, K., \& Zilles, K. (2006). The human inferior parietal cortex: Cytoarchitectonic parcellation and interindividual variability. Neuroimage, 33, 430-448.

Cavanna, A. E., \& Trimble, M. R. (2006). The precuneus: A review of its functional anatomy and behavioural correlates. Brain, 129, 564-583.

Choi, H.-J., Zilles, K., Mohlberg, H., Schleicher, A., Fink, G. R., Armstrong, E., et al. (2006). Cytoarchitectonic identification and probabilistic mapping of two distinct areas within the anterior ventral bank of the human intraparietal sulcus. Journal of Comparative Neurology, 495, 53-69.

Cohen Kadosh, R., Lammertyn, J., \& Izard, V. (2008). Are numbers special? An overview of chronometric, neuroimaging, developmental and comparative studies of magnitude representation. Progress in Neurobiologv, 84, 132-147.

Cohen Kadosh, R., \& Walsh, V. (2009). Numerical representation in the parietal lobes: Abstract or not abstract? The Behavioral and Brain Sciences, 32, 313-373.

Cutini, S., Scarpa, F., Scatturin, P., Dell'acqua, R., \& Zorzi, M. (2012). Number-space interactions in the human parietal cortex: Enlightening the SNARC effect with functional near-infrared spectroscopy. Cerebral Cortex. doi: 10.1093/cercor/bhs321.

de Hevia, M. D., Vallar, G., \& Girelli, L. (2008). Visualizing numbers in the mind's eye: The role of visuo-spatial processes in numerical abilities. Neuroscience and Biobehavioral Reviews, 32, 1361-1372.

Dehaene, S. (1992). Varieties of numerical abilities. Cognition, 44, 1-42.

Dehaene, S. (1997). The number sense. How the mind creates mathematics. New York: Oxford University Press.

Dehaene, S. (2009). Origins of mathematical intuitions: The case of arithmetic. Annals of the New York Academv of Sciences, 1156, 232-259.
Dehaene, S., Bossini, S., \& Giraux, P. (1993). The mental representation of parity and number magnitude. Journal of Experimental Psvchologv: General, 122, 371-396.

Dehaene, S., Piazza, M., Pinel, P., \& Cohen, L. (2003). Three parietal circuits for number processing. Cognitive Neuropsychology, 20, 487-506.

Delazer, M., Domahs, F., Bartha, L., Brenneis, C., Lochy, A., Trieb, T., et al. (2003). Learning complex arithmetic-An fMRI study. Brain Research. Cognitive Brain Research, 18, 76-88.

Eickhoff, S., Stephan, K., Mohlberg, H., \& Grefkes, C. (2005). A new SPM toolbox for combining probabilistic cytoarchitectonic maps and functional imaging data. Neuroimage, 25, 1325-1335.

Fias, W., Brysbaert, F., Geypens, F., \& Ydewalle, G. (1996) The importance of magnitude information in numerical processing: Evidence from the SNARC effect. Mathematical Cognition, 2, 95-110.

Fischer, M. (2006). The future for Snarc could be stark.... Cortex, 42, 1066-1068.

Fischer, U., Moeller, K., Bientzle, M., Cress, U., \& Nuerk, H.-C. (2011). Sensori-motor spatial training of number magnitude representation. Psychonomic Bulletin E Review, 18, 177-183.

Gerstmann, J. (1940). Syndrome of finger agnosia, disorientation for right and left, agraphia and acalculia-Local diagnostic value. Archives of Neurological Psychiatry, 44, 398-408.

Gevers, W., Reynvoet, B., \& Fias, W. (2003). The mental representation of ordinal sequences is spatially organized. Cognition, 87, B87-B95.

Gevers, W., Reynvoet, B., \& Fias, W. (2004). The mental representation of ordinal sequences is spatially organized: Evidence from days of the week. Cortex, 40, 171-172.

Göbel, S., Walsh, V., \& Rushworth, M. F. S. (2001). The mental number line and the human angular gyrus. Neuroimage, 14 , $1278-1289$

Good, C. D., Johnsrude, I. S., Ashburner, J., Henson, R. N., Friston, K. J., \& Frackowiak, R. S. (2001). A voxel-based morphometric study of ageing in 465 normal adult human brains. Neuroimage, 14, 21-36.

Grabner, R. H., Ansari, D., Koschutnig, K., Reishofer, G., Ebner, F., \& Neuper, C. (2009). To retrieve or to calculate? Left angular gyrus mediates the retrieval of arithmetic facts during problem solving. Neuropsvchologia. 47, 604-608.

Grabner, R. H., Ansari, D., Reishofer, G., Stern, E., Ebner, F., \& Neuper, C. (2007). Individual differences in mathematical competence predict parietal brain activation during mental calculation. Neuroimage, 38, 346-356.

Holloway, I. D., Price, G. R., \& Ansari, D. (2010). Common and segregated neural pathways for the processing of symbolic and nonsymbolic numerical magnitude: An fMRI study. Neuroimage, 49, 1006-1017.

Hubbard, E. M., Piazza, M., Pinel, P., \& Dehaene, S. (2005). Interactions between number and space in parietal cortex. Nature Reviews Neuroscience, 6, 435-448.

Ischebeck, A., Zamarian, L., Siedentopf, C., Koppelstätter, F., Benke, T., Felber, S., et al. (2006). How specifically do we learn? Imaging the learning of multiplication and subtraction. Neuroimage, 30, 1365-1375.

Jones, E. G., Coulter, J. D., \& Hendry, S. H. (1987). Intracortical connectivity of architectonic fields in the somatic sensory, motor and parietal cortex of monkeys. Journal of Comparative Neurology, 181, 291-347.

Kanai, R., Feilden, T., Firth, C., \& Rees, G. (2011). Political orientations are correlated with brain structure in young adults. Current Biology, 21, 677-680.

Krause, F., Bekkering, H., \& Lindemann, O. (2013). A feeling for numbers: Shared metric for symbolic and tactile numerosities. Frontiers in Psychology, 4, 7. 
Lacquaniti, F., Guigon, E., Bianchi, L., Ferraina, S., \& Caminiti, R. (1995). Representing spatial information for limb movement: Role of area 5 in the monkey. Cerebral Cortex, 5, 391-409.

Lindemann, O., Abolafia, J. M., Girardi, G., \& Bekkering, H. (2007). Getting a grip on numbers: Numerical magnitude priming in object grasping. Journal of Experimental Psychologv: Human Perception and Performance, 33, 1400-1409.

Lindemann, O., Rueschemeyer, S.-A., \& Bekkering, H. (2009). Symbols in numbers: From numerals to magnitude information. Commentary. Behavioural and Brain Sciences, 32, 341-342

Maguire, E. A., Gadian, D. G., Johnsrude, I. S., Good, C. D., Ashburner, J., Frackowiak, R. S., et al. (2000). Navigationrelated structural change in the hippocampi of taxi drivers. Proceedings of the National Academv of Sciences, U.S.A., 97, 4398-4403.

Mechelli, A., Crinion, J. T., Noppeney, U., O’Doherty, J., Ashburner, J., Frackowiak, R. S., et al. (2004). Neurolinguistics: Structural plasticity in the bilingual brain. Nature, 431, 757.

Mechelli, A., Price, C. J., Friston, K. J., \& Ashburner, J. (2005). Voxel-based morphometry of the human brain: Methods and applications. Current Medical Imasing Reviews, 1, 105-113.

Mountcastle, V. B., Lynch, J. C., Georgopoulos, A., Sakata, H., \& Acuna, C. (1975). Posterior parietal association cortex of the monkey: Command functions for operations within extrapersonal space. Journal of Neurophysiology, 38, 871-908.

Moyer, R. S., \& Landauer, T. K. (1967). Time required for judgements of numerical inequality. Nature, 215, 1519-1520.

Oliveri, M., Vicario, C. M., Salerno, S., Koch, G., Turriziani, P., Mangano, R., et al. (2008). Perceiving numbers alters time perception. Neuroscience Letters, 438, 308-311.

Poser, B. A., Versluis, M. J., Hoogduin, J. M., \& Norris, D. G. (2006). BOLD contrast sensitivity enhancement and artifact reduction with multiecho EPI: Parallel-acquired inhomogeneity-desensitized fMRI. Magnetic Resonance in Medicine, 55, 1227-1235.

Price, G. R., \& Ansari, D. (2011). Symbol processing in the left angular gyrus: Evidence from passive perception of digits Neuroimage, 57, 1205-1211.
Rusconi, E., Walsh, V., \& Butterworth, B. (2005). Dexterity with numbers: rTMS over left angular gyrus disrupts finger gnosis and number processing. Neuropsvchologia. 43 1609-1624.

Santens, S., \& Gevers, W. (2008). The SNARC effect does not imply a mental number line. Cognition, 108, 263-270.

Scheperjans, F., Eickhoff, S. B., Hömke, L., Mohlberg, H., Hermann, K., Amunts, K., et al. (2008). Probabilistic maps, morphometry, and variability of cytoarchitectonic areas in the human superior parietal cortex. Cerebral Cortex, 18, 2141-2157.

Scheperjans, F., Grefkes, C., Palomero-Gallagher, N., Schleicher, A., \& Zilles, K. (2005). Subdivisions of human parietal area 5 revealed by quantitative receptor autoradiography: A parietal region between motor, somatosensory, and cingulate cortical areas. Neuroimage 25, 975-992.

Scheperjans, F., Hermann, K., Eickhoff, S. B., Amunts, K., Schleicher, A., \& Zilles, K. (2008). Observer-independent cytoarchitectonic mapping of the human superior parietal cortex. Cerebral Cortex, 18, 846-867.

Tzelgov, J., Meyer, J., \& Henik, A. (1992). Automatic and intentional processing of numerical information. Lournal of Experimental Psychology: Learning. Memory. and Cognition $18,166$.

Vierck, E., \& Kiesel, A. (2010). Congruency effects between number magnitude and response force. Iournal of Experimental Psychologv: Learning. Memorv. and Cognition. 36, 204-209.

Walsh, V. (2003). A theory of magnitude: Common cortical metrics of time, space and quantity. Trends in Cognitive Sciences, 7, 483-488.

Wood, G., Nuerk, H., \& Willmes, K. (2008). On the cognitive link between space and number: A meta-analysis of the SNARC effect. Psychology Science, 50, 489-525.

Wu, S. S., Chang, T. T., Majid, A., Caspers, S., Eickhoff, S. B., \& Menon, V. (2009). Functional heterogeneity of inferior parietal cortex during mathematical cognition assessed with cytoarchitectonic probability maps. Cerebral Cortex, 19, $2930-2945$. 
This article has been cited by:

1. Anna Milena Dubischar-Krivec, Sven Bölte, Christoph Braun, Fritz Poustka, Niels Birbaumer, Nicola Neumann. 2014. Neural mechanisms of savant calendar calculating in autism: An MEG-study of few single cases. Brain and Cognition 90, 157-164. [CrossRef]

2. Xiuqin Jia, Peipeng Liang, Lin Shi, Defeng Wang, Kuncheng Li. 2014. Prefrontal and parietal activity is modulated by the rule complexity of inductive reasoning and can be predicted by a cognitive model. Neuropsychologia . [CrossRef]

3. Jenny Gu, Ryota Kanai. 2014. What contributes to individual differences in brain structure?. Frontiers in Human Neuroscience 8. . [CrossRef] 